\title{
Analytical Calculation of Power Flow in Three Coupled Oscillators and its Application to One Dimensional Crystal
}

\author{
Chifu E. Ndikilar, Sofwan I. Saleh, Hafeez Y. Hafeez and Lawan S. Taura
}

\section{ABSTRACT}

The motion of a system consisting of three coupled oscillators of three masses attached together by four springs is studied analytically. The system is used as a model to describe the interactions between atoms in a one dimensional crystal with spring-like forces under white noise excitations. Two different cases are considered and the frequencies of oscillations are obtained as well as the equations of motion. The equations of motions are used to determine the power flow in the systems. The power flow determined is used to describe the effects of substitution impurities in a crystal. The power flow of the two systems studied decreases exponentially with increase in frequency to an asymptotic value.

Keywords: coupled oscillators, power flow, crystal, white noise
Published Online: February 22,2020

ISSN: $2684-4451$

DOI : $10.24018 / 2020.2 .1 .4$

Chifu E. Ndikilar *

Department of Physics, Federal University Dutse, Jigawa State, Nigeria .(e-mail: ebenechifu@yahoo.com) Sofwan I. Saleh

Department of Physics, Sule Lamido University Kafin-Hausa, Jigawa State, Nigeria.

Hafeez Y. Hafeez

Department of Physics, Federal University Dutse, Jigawa State, Nigeria. (e-mail:

hafeezyusufhafeez@gmail.com)

Lawan S. Taura

Department of Physics, Sule Lamido University Kafin-Hausa, Jigawa State,

Nigeria. (e-mail: 1staura@yahoo.com)

*Corresponding Author

\section{INTRODUCTION}

Many important physical systems involve coupled oscillators. Coupled oscillators are oscillators connected in such a way that energy can be transferred between them. The motion of coupled oscillators can be complex and does not have to be periodic. However, when the oscillators carry out complex motion, we can find a coordinate frame in which each oscillator oscillates with a very well defined frequency [1]. Consequently, a coupled system is a system of mutually interacting particles where by the motion of a given particle is influenced by the motion of all other particles in the system and the overall motion of the system is the resultant of the motion of all individual particles. Restricting the amplitude of oscillation to be small the entire system develops superposition of distinct modes of motion, referred to as normal modes of motion. In each of such modes, the system oscillates with its own characteristic frequency and assumes a configuration peculiar to the frequency [2].

In the last few years there has been an increasing interest in the statistical behavior of a set of coupled oscillators. Chemical reaction rates may be controlled by the rate of energy flow between the vibrational modes of molecules. Wave interactions in fluids may also be explained by the different rates of inter-modal energy transfer. An important engineering application is the transmission of noise energy between coupled structures [3].

Coupling between oscillators was first studied by Huygens in 1673 who noticed that pendulum clocks in the same room became synchronized. Electrical circuits are man-made systems for handling and transport of energy. The electrical world may be coupled to the mechanical world by means of flux (generators, motors). The electrical world may be coupled to the chemical world by means of charge (batteries) [4].

Coupled and synchronized oscillators are significant in electronics also. The dynamics of a phase-locked loop can be highly nonlinear and are not fully understood. For example, chaotic dynamics and locking region hysteresis are possible. Commonly, only a simplified linear model of the dynamics is used. Coupled oscillators also occur in biological systems. One early use of mathematics to model biological systems was Van der Pol's use in 1928 of a driven Van der Pol oscillator to explain some normal and pathological, rhythms of the heart. However, Van der Pol devised his differential equation to model an electronic oscillator based on the triode valve [5].

A crystal is also a good example of a system that can be 
described in terms of coupled oscillations. The atoms oscillate around their equilibrium positions, and the interaction between the atoms is responsible for the coupling, based on the assumption that the forces involved are springlike forces i.e. the magnitude of the force is proportional to the magnitude of the displacement from equilibrium [1]. A crystal is a solid material whose constituents (such as atoms, molecules, or ions) are arranged in a highly ordered microscopic structure, forming a crystal lattice that extends in all directions [6]. Remarkably, many of the essentials of crystal Physics can be obtained without reference to quantum mechanics, by thinking of a crystal as an orderly assembly of Newtonian point particles connected to one another (usually only to near neighbors) by springs (see Fig. 1).

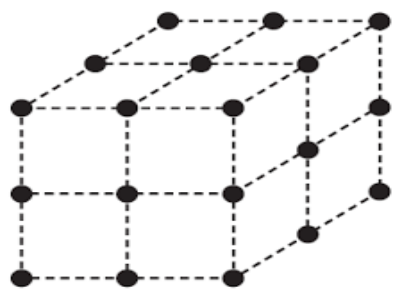

Fig. 1: three dimensional crystal

Chifu et. al. calculated the power flow between two weakly coupled pendula analytically and applied to explain the behavior of an optical switch. The power flow under white noise random excitation between the pendula in each system is calculated. The result showed that the net power flow corresponds to the system with identical pendula and for nonidentical pendula, the phase constants are not equal and there will be no net power flow between the waveguides [7].

In this research work, the system considered represents a one dimensional crystal consisting of three atoms with identical masses connected to each other and to the walls of the crystal based on the assumption that the atoms are connected together by a force similar to the force on a spring where by the restoring force F obeys Hooke's law under white noise excitation. Consequently, such a system is a simplified model of a one dimensional crystal and hence can be solved using methods of analytical mechanics.

\section{ANALYTICAL METHODS}

\section{A. Description of the System}

The system considered consist of three particles of masses $\mathrm{m} 1, \mathrm{~m} 2$ and $\mathrm{m} 3$ attached to four horizontal identical spiral springs each of length 1 and force constant $\mathrm{k}$ such that the masses are free to oscillate in a horizontal straight line (Fig. 2).

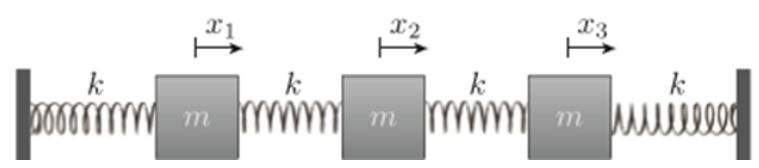

Fig. 2: a system of three masses and four springs (horizontally aligned).

The system has three degrees of freedom $\mathrm{q}_{1}, \mathrm{q}_{2}$ and $\mathrm{q}_{3}$ (displacement of the rest masses from equilibrium position). The kinetic energy of the system is given by [2]:
$T=\frac{1}{2} m_{1} \dot{q}_{1}^{2}+\frac{1}{2} m_{2} \dot{q}_{2}^{2}+\frac{1}{2} m_{3} \dot{q}_{3}^{2}$

If $\mathrm{m}_{1}=\mathrm{m}_{2}=\mathrm{m}$, while $\mathrm{m}_{3}=\mathrm{nm}$ then

$T=\frac{1}{2} m \dot{q}_{1}^{2}+\frac{1}{2} n m \dot{q}_{2}^{2}+\frac{1}{2} m \dot{q}_{3}^{2}$

In matrix form

$2 T=\left(\begin{array}{lll}\dot{q}_{1} & \dot{q}_{2} & \dot{q}_{3}\end{array}\right)\left(\begin{array}{ccc}m & 0 & 0 \\ 0 & n m & 0 \\ 0 & 0 & m\end{array}\right)\left(\begin{array}{l}\dot{q}_{1} \\ \dot{q}_{2} \\ \dot{q}_{3}\end{array}\right)$

Therefore, we can define:

$$
T_{i j}=\left(\begin{array}{ccc}
m & 0 & 0 \\
0 & n m & 0 \\
0 & 0 & m
\end{array}\right)
$$

The potential energy of the system is given by:

$$
V=\frac{1}{2} k q_{1}^{2}+\frac{1}{2} k\left(q_{2}-q_{1}\right)^{2}+\frac{1}{2} k\left(q_{3}-q_{2}\right)^{2}+\frac{1}{2} k q_{3}^{2}
$$

The matrix form is given by:

$$
2 V=\left(\begin{array}{lll}
q_{1} & q_{2} & q_{3}
\end{array}\right)\left(\begin{array}{ccc}
2 k & -k & 0 \\
-k & 2 k & -k \\
0 & -k & 2 k
\end{array}\right)\left(\begin{array}{l}
q_{1} \\
q_{2} \\
q_{3}
\end{array}\right)
$$

with

$$
V_{i j}=\left(\begin{array}{ccc}
2 k & -k & 0 \\
-k & 2 k & -k \\
0 & -k & 2 k
\end{array}\right)
$$

\section{B. Derivation of Frequencies of Oscillations}

The kinetic energy, potential energy and the secular equation are used to determine the frequencies of the system for two different cases. The secular equation is given as [2]

$$
\left|V_{i j}-\omega_{i}^{2} T_{i j}\right|=0
$$

Now, substituting and from (4) and (7) in (8) gives:

$$
\left|\begin{array}{ccc}
2 k-\omega_{i}^{2} m & -k & 0 \\
-k & 2 k-\omega_{i}^{2} n m & -k \\
0 & -k & 2 k-\omega_{i}^{2} m
\end{array}\right|=0
$$

Solving for gives:

$$
\begin{aligned}
& \omega_{1}^{2}=\frac{2 k}{m}, \omega_{2}^{2}=\frac{k}{n m}\left[(1+n)+\left(1+n^{2}\right)^{\frac{1}{2}}\right] \\
& \omega_{3}^{2}=\frac{k}{n m}\left[(1+n)-\left(1+n^{2}\right)^{\frac{1}{2}}\right]
\end{aligned}
$$

Where $\mathrm{n}$ can be any positive integer

If $n=1$ then the three masses will be identical and the frequencies of vibration will be given as:

$$
\omega_{1}^{2}=\frac{2 k}{m}, \omega_{2}^{2}=\frac{k(2+\sqrt{2})}{m}, \omega_{3}^{2}=\frac{k(2-\sqrt{2})}{m}
$$


Suppose $n=2$ then the middle mass is different from the two end masses, so the new frequencies of oscillation will be given:

$$
\omega_{1}^{2}=\frac{2 k}{m}, \quad \omega_{2}^{2}=\frac{k(3+\sqrt{5})}{2 m}, \omega_{3}^{2}=\frac{k(3-\sqrt{5})}{2 m}
$$

\section{Calculation of Power Flow}

The system has three degrees of freedom and the normal coordinates are given as [2]:

$$
\left(\begin{array}{l}
q_{1} \\
q_{2} \\
q_{3}
\end{array}\right)=\left(\begin{array}{lll}
a_{11} & a_{21} & a_{31} \\
a_{12} & a_{22} & a_{32} \\
a_{13} & a_{23} & a_{33}
\end{array}\right)\left(\begin{array}{l}
\eta_{1} \\
\eta_{2} \\
\eta_{3}
\end{array}\right)
$$

where are components of an Eigen vector corresponding to a particular frequency, such that:

$$
\sum_{i}\left(V_{i j}-\omega_{i}^{2} T_{i j}\right) a_{i j}=0
$$

Then the value of is determined using:

$$
a^{T} T a=1
$$

The equations of motion in normalized coordinates are obtained using

$$
\ddot{\eta}_{n}+\omega_{n}^{2} \eta_{n}=0
$$

Equation (13) is used to transform the normalized coordinates into the equations of motion in generalized coordinates.

For two coupled oscillators, the equations of motion can be written in the form:

$$
\begin{aligned}
& \ddot{q}_{1}+2 \beta_{1} \Omega_{1} \dot{q}_{1}+\Omega_{1}^{2} q_{1}=\frac{f_{1}(t)}{m_{1}}+\frac{\varepsilon \gamma}{m_{1}} q_{1} \\
& \ddot{q}_{2}+2 \beta_{2} \Omega_{2} \dot{q}_{2}+\Omega_{2}^{2} q_{2}=\frac{f_{2}(t)}{m_{2}}+\frac{\varepsilon \gamma}{m_{2}} q_{2}
\end{aligned}
$$

with

$$
C_{i}=2 m_{i} \beta_{i} \Omega_{i}
$$

The rate of energy flow between two coupled oscillators when the excitation of each is an independent source of stationary white noise is given by [3]:

$$
\Pi_{i j}=\frac{\varepsilon^{2} \sigma^{2} U}{m_{i} m_{j}}\left[\frac{2\left(\beta_{i} \Omega_{i}+\beta_{j} \Omega_{j}\right)}{\left\{\left(\beta_{i} \Omega_{i}+\beta_{j} \Omega_{j}\right)^{2}+\left(p_{i}-p_{j}\right)^{2}\right\}\left\{\left(\beta_{i} \Omega_{i}+\beta_{j} \Omega_{j}\right)^{2}+\left(p_{i}+p_{j}\right)^{2}\right\}}\right]
$$

Equation (20) represent the power dissipated by the damping of the oscillator with

$$
p_{i}=\Omega_{i} \sqrt{1-\beta_{i}^{2}}, p_{j}=\Omega_{j} \sqrt{1-\beta_{j}^{2}}
$$

where $\varepsilon^{2}, \sigma^{2}$ are coupling parameters, $C_{i}$ is the damping of the $\mathrm{i}^{\text {th }}$ oscillator, $\mathrm{U}$ is the energy difference of the two oscillators and $\beta_{\mathrm{i}}, \beta_{\mathrm{j}}, \Omega_{\mathrm{i}}, \Omega_{\mathrm{j}}$, mi and $\mathrm{m}_{\mathrm{j}}$ are the damping ratios, natural blocked frequencies and the masses of the $\mathrm{i}^{\text {th }}$ and $\mathrm{j}^{\text {th }}$ oscillators respectively.

The power flow in equation (20) can be related to the damping of the oscillator by the equation:

$$
\Pi_{i}=c_{i} E\left[\dot{q}_{i}^{2}\right]
$$

where $\mathrm{E}$ is the statistical average. The kinetic energy of an oscillator is given by:

$$
T_{i}=\frac{1}{2} m_{i} E\left[\dot{q}_{i}^{2}\right]
$$

If the blocked kinetic energy of the first oscillator is defined as its energy when the second oscillator is clamped, then:

$$
T_{1 B}=\frac{1}{2} m_{1} E\left[\dot{q}_{10}^{2}\right]=\frac{1}{2} U_{10}
$$

The mean kinetic energy of the second oscillator as a function of the natural frequencies for the case when only the first oscillator is excited with white noise is given by [3]:

$$
\frac{T_{2}}{T_{1 B}}=\frac{C\left\{1+\frac{\beta_{1} \Omega_{1}}{\beta_{2} \Omega_{2}}\right\}\left(\frac{\Omega_{2}}{\Omega_{1}}\right)^{2}}{\left\{\left(\beta_{1}+\frac{\beta_{2} \Omega_{2}}{\Omega_{1}}\right)^{2}+\left(\frac{p_{1}-p_{2}}{\Omega_{1}}\right)^{2}\right\}\left\{\left(\beta_{1}+\frac{\beta_{2} \Omega_{2}}{\Omega_{1}}\right)^{2}+\left(\frac{p_{1}+p_{2}}{\Omega_{1}}\right)^{2}\right\}}
$$

where $\mathrm{C}$ is the coupling coefficient.

\section{RESULTS AND DISCUSSIONS}

\section{A. System of Identical Masses}

For this system, equation (14) gives:

$$
\left(\begin{array}{lrr}
2 k-\omega_{i}^{2} m & -k & 0 \\
-k & 2 k-\omega_{i}^{2} m & -k \\
0 & -k & 2 k-\omega_{i}^{2} m
\end{array}\right)\left(\begin{array}{l}
a_{i j} \\
a_{i j} \\
a_{i j}
\end{array}\right)=0
$$

For from equation (11), equation (26) becomes

$$
\left(\begin{array}{ccc}
0 & -k & 0 \\
-k & 0 & -k \\
0 & -k & 0
\end{array}\right)\left(\begin{array}{l}
a_{11} \\
a_{12} \\
a_{13}
\end{array}\right)=0
$$

and hence and the normal coordinate corresponding to the frequency is:

$$
\left(\begin{array}{l}
a_{11} \\
a_{12} \\
a_{13}
\end{array}\right)=\left(\begin{array}{l}
\alpha \\
0 \\
-\alpha
\end{array}\right)
$$

Similarly, for and the normal coordinates are obtained respectively as

$$
\begin{aligned}
&\left(\begin{array}{l}
a_{21} \\
a_{22} \\
a_{23}
\end{array}\right)=\left(\begin{array}{c}
\beta \\
-\beta \sqrt{2} \\
\beta
\end{array}\right) \\
&\left(\begin{array}{l}
a_{31} \\
a_{32} \\
a_{33}
\end{array}\right)=\left(\begin{array}{c}
\gamma \\
\gamma \sqrt{2} \\
\gamma
\end{array}\right)
\end{aligned}
$$

and hence

$$
a=\left(\begin{array}{ccc}
\alpha & \beta & \gamma \\
0 & -\beta \sqrt{2} & \gamma \sqrt{2} \\
-\alpha & \beta & \gamma
\end{array}\right)
$$

Using equations (15) yields:

$$
\alpha=\frac{1}{\sqrt{2 m}}, \quad \beta=\gamma=\frac{1}{2 \sqrt{m}}
$$

Thus, equation (13) can be written as: 


$$
\left(\begin{array}{l}
q_{1} \\
q_{2} \\
q_{3}
\end{array}\right)=\left(\begin{array}{ccc}
\frac{1}{\sqrt{2 m}} & \frac{1}{2 \sqrt{m}} & \frac{1}{2 \sqrt{m}} \\
0 & -\frac{1}{\sqrt{2 m}} & \frac{1}{\sqrt{2 m}} \\
-\frac{1}{\sqrt{2 m}} & \frac{1}{2 \sqrt{m}} & \frac{1}{2 \sqrt{m}}
\end{array}\right)\left(\begin{array}{l}
\eta_{1} \\
\eta_{2} \\
\eta_{3}
\end{array}\right)
$$

and simplifying gives:

$$
\begin{aligned}
& \eta_{1}=\sqrt{\frac{m}{2}}\left(q_{1}-q_{3}\right), \eta_{2}=\frac{\sqrt{m}}{2}\left(q_{1}+q_{3}-\sqrt{2} q_{2}\right), \\
& \eta_{3}=\frac{\sqrt{m}}{2}\left(q_{1}+q_{3}+\sqrt{2} q_{2}\right)
\end{aligned}
$$

The derived equations of motion in generalized coordinates after the introduction of the excitation due to the first, second and third oscillators are given respectively as:

$$
\begin{array}{r}
m \ddot{q}_{1}+c_{1} \dot{q}_{1}+2 k q_{1}=f_{1}(t)+k q_{2} \\
m \ddot{q}_{2}+c_{2} \dot{q}_{2}+2 k q_{2}=f_{2}(t)+k\left(q_{1}+q_{3}\right) \\
m \ddot{q}_{3}+c_{3} \dot{q}_{3}+2 k q_{3}=f_{3}(t)+k q_{2}
\end{array}
$$

If $f_{1}(t), f_{2}(t)$ and $f_{3}(t)$ are the independent sources of stationary Gaussian white noise excitation and we consider the excitation of the middle oscillator to be zero i.e. it is not excited, while the two end atoms are excited, then the energy of the middle oscillator arises from power flow between the first and third oscillators to the middle oscillator.

The total power of the middle oscillator is equal to the sum of the individual power flow due to the first and the third oscillator respectively as:

$$
\Pi_{2}=\Pi_{12}+\Pi_{32}
$$

Since the two end oscillators exhibit same vibrations then, the power flow from the first oscillator is equal to that from the third and hence:

$$
\Pi_{2}=2 \Pi_{12}
$$

Equations (35), (36) and (37) can be re-written as:

$$
\begin{array}{r}
\ddot{q}_{1}+2 \beta_{1} \Omega_{1} \dot{q}_{1}+\Omega_{1}^{2} q_{1}=\frac{f_{1}(t)}{m}+\frac{k}{m} q \\
\ddot{q}_{2}+2 \beta_{2} \Omega_{2} \dot{q}_{2}+\Omega_{2}^{2} q_{2}=\frac{f_{2}(t)}{m}+\frac{k}{m}\left(q_{1}+q_{3}\right) \\
\ddot{q}_{3}+2 \beta_{3} \Omega_{3} \dot{q}_{3}+\Omega_{3}^{2} q_{3}=\frac{f_{3}(t)}{m}+\frac{k}{m} q_{2}
\end{array}
$$

Thus, the power flow can be written as:

$$
\Pi_{2}=\frac{2 k^{2} U}{m^{2}}\left[\frac{2\left(\beta_{1} \Omega_{1}+\beta_{2} \Omega_{2}\right)}{\left\{\left(\beta_{1} \Omega_{1}+\beta_{2} \Omega_{2}\right)^{2}+\left(p_{1}-p_{2}\right)^{2}\right\}\left\{\left(\beta_{1} \Omega_{1}+\beta_{2} \Omega_{2}\right)^{2}+\left(p_{1}+p_{2}\right)^{2}\right\}}\right]
$$

But in this case, $\beta_{1}=\beta_{2}, \Omega_{1}=\Omega_{2}, p_{1}=p_{2}$ and thus (43) reduces to:

$$
\Pi_{2}=C\left[\frac{(2 \beta \Omega)}{\left\{(2 \beta \Omega)^{2}\right\}\left\{(2 \beta \Omega)^{2}+(2 p)^{2}\right\}}\right]
$$

Equation (44) represents the power flow of the second oscillator in a system of three identical masses and four springs. It is illustrated with the computed parameters in Table I and Fig. 3

TABLE I: CALCULATED POWER FLOW AND CORRESPONDING

\begin{tabular}{|l|l|l|l|}
\hline \multicolumn{1}{|c|}{ FREQUENCIES } \\
\hline$\Omega^{*} 10^{12}(\mathrm{~Hz})$ & $\mathrm{P} * 10^{12}(\mathrm{~Hz})$ & $\mathrm{U}^{*} 10^{23}(\mathrm{~J})$ & $\Pi^{*} 10^{10}(\mathrm{~W})$ \\
\hline 1.73 & 1.73 & 0.2 & 1.7 \\
\hline 3.46 & 3.46 & 0.8 & 0.87 \\
\hline 6.92 & 6.92 & 3.2 & 0.45 \\
\hline 13.84 & 13.84 & 12.7 & 0.22 \\
\hline 27.68 & 27.68 & 50.9 & 0.11 \\
\hline
\end{tabular}

$\mathrm{B}=0.01 A N D \mathrm{C}=3.62 \times 10^{49}[6]$

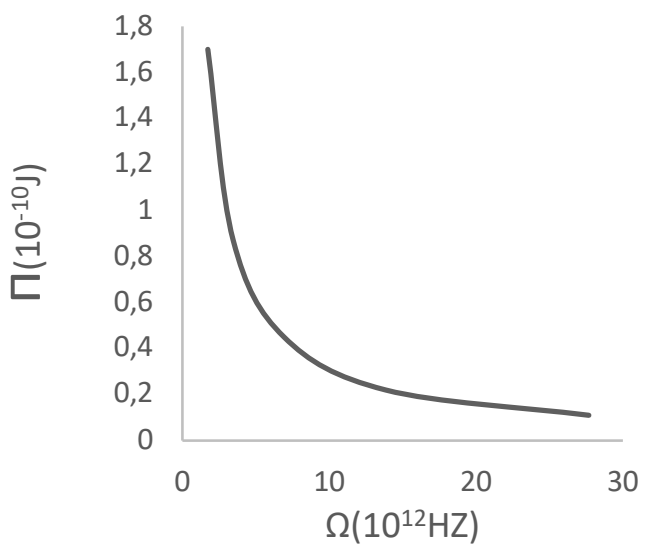

Fig. 3. Power flow against frequency for identical masses.

In Fig. 3, the power flow of the second oscillator decreases exponentially with increase in frequency to an asymptotic value (zero).

The corresponding kinetic energy is obtained as:

$$
T_{2}=\frac{2 k^{2} U}{m^{2} \Omega_{1}^{2} \Omega_{2}^{2}}\left[\frac{\left\{1+\frac{\beta_{1} \Omega_{2}}{\beta_{2} \Omega_{2}}\right\}\left(\frac{\Omega_{2}}{\Omega_{1}}\right)^{2}}{\left\{\left(\beta_{1}+\frac{\beta_{1} \Omega_{2}}{\Omega_{1}}\right)^{2}+\left(\frac{p_{1}-p_{2}}{\Omega_{1}}\right)^{2}\right\}\left\{\left(\beta_{1}+\frac{\beta_{1} \Omega_{2}}{\Omega_{1}}\right)^{2}+\left(\frac{p_{1}+p_{2}}{\Omega_{1}}\right)^{2}\right\}}\right]
$$

and thus using equation (25) we get:

$$
\frac{T_{2}}{T_{1 B}}=\frac{4 k^{2}}{m^{2} \Omega_{1}^{2} \Omega_{2}^{2}}\left[\frac{\left\{1+\frac{\beta_{1} \Omega_{1}}{\beta_{2} \Omega_{2}}\right\}\left(\frac{\Omega_{2}}{\Omega_{1}}\right)^{2}}{\left\{\left(\beta_{1}+\frac{\beta_{2} \Omega_{2}}{\Omega_{1}}\right)^{2}+\left(\frac{p_{1}-p_{2}}{\Omega_{1}}\right)^{2}\right\}\left\{\left(\beta_{1}+\frac{\beta_{2} \Omega_{2}}{\Omega_{1}}\right)^{2}+\left(\frac{p_{1}+p_{2}}{\Omega_{1}}\right)^{2}\right\}}\right]
$$


(46)

with the coupling coefficient $(\mathrm{C})$ defined as:

$$
C=\frac{4 k^{2}}{m^{2} \Omega_{1}^{2} \Omega_{2}^{2}}
$$

Equation (46) represent the ratio of the kinetic energy of the second oscillator to the blocked kinetic energy of the first oscillator of the system and is illustrated in Table II and Fig. 4.

TABLE II: THE RATIO OF KINETIC ENERGIES AND THEIR CORRESPONDING FREQUENCY.

\begin{tabular}{|c|c|c|c|c|c|c|}
\hline $\begin{array}{l}\Omega_{1} * \\
10^{12} \\
(\mathrm{~Hz})\end{array}$ & $\begin{array}{l}\mathrm{P}_{1}^{*} \\
10^{12} \\
(\mathrm{~Hz})\end{array}$ & $\begin{array}{l}\Omega_{2}{ }^{*} \\
10^{12} \\
(\mathrm{~Hz})\end{array}$ & $\begin{array}{l}\mathrm{P}_{2}{ }^{*} \\
10^{12} \\
(\mathrm{~Hz})\end{array}$ & $\mathrm{C}$ & $\begin{array}{l}\Omega_{2} / \\
\Omega_{1}\end{array}$ & $\begin{array}{l}\mathrm{T}_{2} / \\
\mathrm{T}_{1}\end{array}$ \\
\hline 8.65 & 8.65 & 4.33 & 4.33 & $\begin{array}{l}0.02 \\
6\end{array}$ & 0.5 & $\begin{array}{l}0.03 \\
4\end{array}$ \\
\hline 6.49 & 6.49 & 5.22 & 5.22 & $\begin{array}{l}0.03 \\
2\end{array}$ & 0.8 & 0.35 \\
\hline 5.85 & 5.85 & 5.85 & 5.85 & $\begin{array}{l}0.03 \\
1\end{array}$ & 1 & 38.8 \\
\hline 5.20 & 5.20 & 6.49 & 6.49 & $\begin{array}{l}0.03 \\
2\end{array}$ & 1.2 & 0.25 \\
\hline 5.77 & 5.77 & 8.65 & 8.65 & $\begin{array}{l}0.01 \\
4\end{array}$ & 1.5 & $\begin{array}{l}0.00 \\
3\end{array}$ \\
\hline
\end{tabular}

$\beta_{1}=\beta_{2}=0.01$

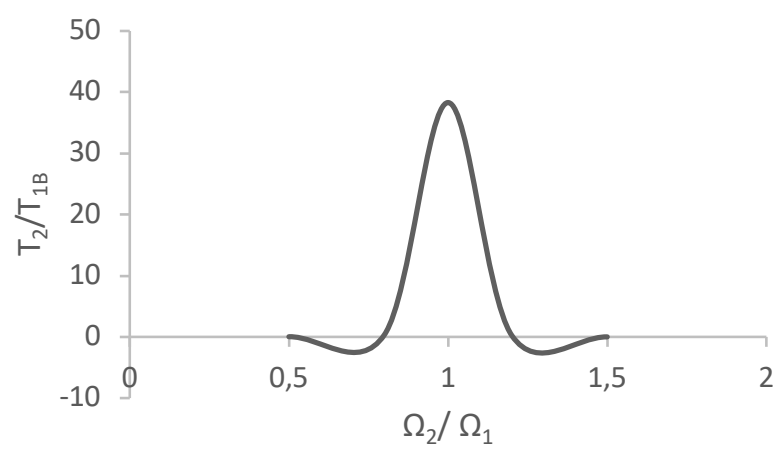

Fig. 4. Ratios of the kinetic energy against frequencies.

The ratio of the kinetic energy has a maximum value when the ratio of frequencies is equal to unity.

\section{B. System of Non-Identical Masses}

The normal coordinate corresponding respectively to the frequencies $\omega_{1}, \omega_{2}$ and $\omega_{3}$ for this system are obtained as

$$
\begin{aligned}
& \left(\begin{array}{l}
a_{11} \\
a_{12} \\
a_{13}
\end{array}\right)=\left(\begin{array}{l}
\alpha \\
0 \\
-\alpha
\end{array}\right) \\
& \left(\begin{array}{l}
a_{21} \\
a_{22} \\
a_{23}
\end{array}\right)=\left(\begin{array}{c}
\beta \\
\beta / 2(1-\sqrt{5}) \\
\beta
\end{array}\right)
\end{aligned}
$$

$$
\left(\begin{array}{l}
a_{31} \\
a_{32} \\
a_{33}
\end{array}\right)=\left(\begin{array}{c}
\gamma \\
\gamma / 2(1+\sqrt{5}) \\
\gamma
\end{array}\right)
$$

Thus

$$
a=\left(\begin{array}{ccc}
\alpha & \beta & \gamma \\
0 & \beta / 2(1-\sqrt{5}) & \gamma / 2(1+\sqrt{5}) \\
-\alpha & \beta & \gamma
\end{array}\right)
$$

with $\alpha=\frac{1}{\sqrt{2 m}}, \beta=\frac{1}{(5 m-m \sqrt{5})^{\frac{1}{2}}}, \quad \gamma=\frac{1}{(5 m+m \sqrt{5})^{\frac{1}{2}}}$

The relationship between the normal coordinates and the generalized coordinates is obtained as

$$
\begin{gathered}
\eta_{1}=\sqrt{\frac{m}{2}}\left(q_{1}-q_{3}\right) \\
\eta_{2}=\frac{(1+\sqrt{5})(5 m-m \sqrt{5})^{\frac{1}{2}}}{4 \sqrt{5}}\left(q_{1}+q_{3}-\frac{4 q_{2}}{(1+\sqrt{5})}\right)
\end{gathered}
$$

$\eta_{3}=\frac{(1-\sqrt{5})(5 m+m \sqrt{5})^{\frac{1}{2}}}{4 \sqrt{5}}\left(q_{1}+q_{3}-\frac{4 q_{2}}{(1-\sqrt{5})}\right)$

The equations of motion in generalized coordinates are obtained as

$$
\begin{aligned}
& m \ddot{q}_{1}+c_{1} \dot{q}_{1}+2 k q_{1}=f_{1}(t)+k q_{2} \\
& m \ddot{q}_{2}+c_{2} \dot{q}_{2}+k q_{2}=f_{2}(t)+\frac{k}{2}\left(q_{1}+q_{3}\right) \\
& m \ddot{q}_{3}+c_{3} \dot{q}_{3}+2 k q_{3}=f_{3}(t)+k q_{2}
\end{aligned}
$$

which can be written equivalently as

$$
\begin{aligned}
& \ddot{q}_{1}+2 \beta_{1} \Omega_{1} \dot{q}_{1}+\Omega_{1}^{2} q_{1}=\frac{f_{1}(t)}{m}+\frac{k}{m} q_{2} \\
& \ddot{q}_{2}+2 \beta_{2} \Omega_{2} \dot{q}_{2}+\Omega_{2}^{2} q_{2}=\frac{f_{2}(t)}{m}+\frac{k}{2 m}\left(q_{1}+q_{3}\right) \\
& \ddot{q}_{3}+2 \beta_{3} \Omega_{3} \dot{q}_{3}+\Omega_{3}^{2} q_{3}=\frac{f_{3}(t)}{m}+\frac{k}{m} q_{2}
\end{aligned}
$$

The power flow to the second oscillator is given as

$\Pi_{2}=\frac{k^{2} U}{m^{2}}\left[\frac{2\left(\beta_{1} \Omega_{1}+\beta_{2} \Omega_{2}\right)}{\left\{\left(\beta_{1} \Omega_{1}+\beta_{2} \Omega_{2}\right)^{2}+\left(p_{1}-p_{2}\right)^{2}\right\}\left\{\left(\beta_{1} \Omega_{1}+\beta_{2} \Omega_{2}\right)^{2}+\left(p_{1}+p_{2}\right)^{2}\right\}}\right]$

But $\Omega_{2}=\frac{\Omega_{1}}{\sqrt{2}}$ and $\beta_{2}=\frac{\sqrt{2} \beta_{1}}{2}$, and hence (61) reduces to

$$
\Pi_{2}=C U\left[\frac{3 \beta_{1} \Omega_{1}}{\left\{\left(\frac{3}{2} \beta_{1} \Omega_{1}\right)^{2}+\left(p_{1}-p_{2}\right)^{2}\right\}\left\{\left(\frac{3}{2} \beta_{1} \Omega_{1}\right)^{2}+\left(p_{1}+p_{2}\right)^{2}\right\}}\right]
$$


(62)

Where $C=\frac{k^{2}}{m^{2}}$

Table III and Fig. 5 represents an illustration of the power flow to the second oscillator.

TABLE III: POWER FLOW TO THE SECOND OSCILLATOR

\begin{tabular}{cccccc}
$\Omega_{1} * 10^{12}$ & $\mathrm{P}_{1} * 10^{12}$ & $\Omega_{2} * 10^{12}$ & $\mathrm{P}_{2} * 10^{12}$ & $\begin{array}{l}\mathrm{U}^{*} 10^{-} \\
23\end{array}$ & $\begin{array}{l}\Pi^{*} 10^{-} \\
13\end{array}$ \\
$(\mathrm{~Hz})$ & $(\mathrm{Hz})$ & $(\mathrm{Hz})$ & $(\mathrm{Hz})$ & & $(\mathrm{J} / \mathrm{s})$ \\
\hline 1.73 & 1.73 & 1.22 & 1.22 & 0.2 & 4.40 \\
3.46 & 3.46 & 2.45 & 2.45 & 0.8 & 2.07 \\
6.92 & 6.91 & 4.79 & 4.79 & 3.2 & 1.10 \\
13.84 & 13.83 & 9.79 & 9.79 & 12.7 & 0.24 \\
27.68 & 27.67 & 19.57 & 19.57 & 50.9 & 0.00 \\
\hline
\end{tabular}

$\beta_{1}=0.01$ and $\beta_{2}=0.007[6]$

Fig. 5: Power flow against frequency.

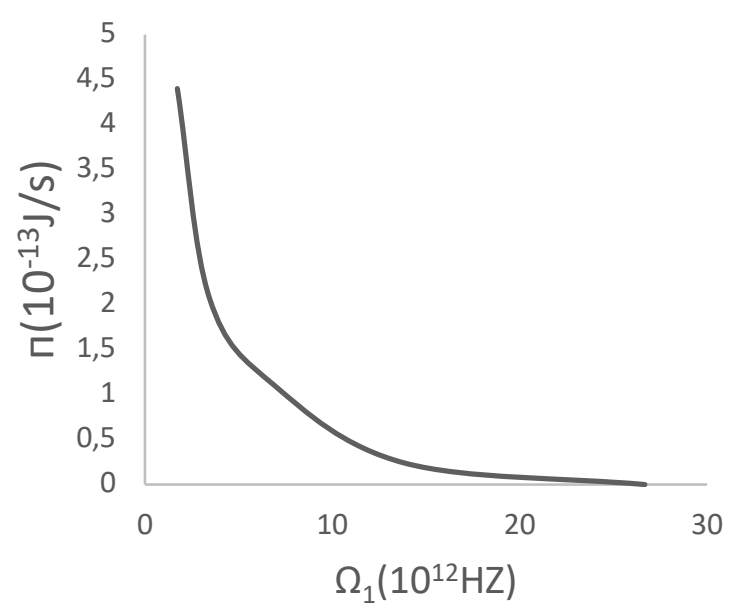

Fig. 5 shows that the power flow of the second oscillator converges exponentially with increase in frequency to a specific limit (zero).

The corresponding kinetic energy is given as

$T_{2}=\frac{k^{2} U}{2 m^{2} \Omega_{1}^{2} \Omega_{2}^{2}}\left[\frac{\left\{1+\frac{\beta_{1} \Omega_{1}}{\beta_{2} \Omega_{2}}\right\}\left(\frac{\Omega_{2}}{\Omega_{1}}\right)^{2}}{\left\{\left(\beta_{1}+\frac{\beta_{1} \Omega_{2}}{\Omega_{1}}\right)^{2}+\left(\frac{p_{1}-p_{2}}{\Omega_{1}}\right)^{2}\right\}\left\{\left(\beta_{1}+\frac{\beta_{1} \Omega_{2}}{\Omega_{1}}\right)^{2}+\left(\frac{p_{1}+p_{2}}{\Omega_{1}}\right)^{2}\right\}}\right]$

The ratio of the kinetic energy of the second oscillator to the blocked kinetic energy of the first oscillator is given as
$\frac{T_{2}}{T_{1 B}}=\frac{k^{2}}{m^{2} \Omega_{1}^{2} \Omega_{2}^{2}}\left[\frac{\left\{1+\frac{\beta_{1} \Omega_{1}}{\beta_{2} \Omega_{2}}\right\}\left(\frac{\Omega_{2}}{\Omega_{1}}\right)^{2}}{\left\{\left(\beta_{1}+\frac{\beta_{1} \Omega_{2}}{\Omega_{1}}\right)^{2}+\left(\frac{p_{1}-p_{2}}{\Omega_{1}}\right)^{2}\right\}\left\{\left(\beta_{1}+\frac{\beta_{1} \Omega_{2}}{\Omega_{1}}\right)^{2}+\left(\frac{p_{1}+p_{2}}{\Omega_{1}}\right)^{2}\right\}}\right]$

where $C=\frac{k^{2}}{m^{2} \Omega_{1}^{2} \Omega_{2}^{2}}$

Table IV represents the relationship between the ratio of kinetic energies and frequencies.

TABLE. IV: THE RATIO OF KINETIC ENERGIES AND THEIR CORRESPONDING FREQUENCY.

\begin{tabular}{|c|c|c|c|c|c|c|}
\hline $\begin{array}{l}\Omega_{1} * 1 \\
0^{12} \\
(\mathrm{~Hz})\end{array}$ & $\begin{array}{l}\mathrm{P}_{1} * 10 \\
12 \\
(\mathrm{~Hz})\end{array}$ & $\begin{array}{l}\Omega_{2} * 10^{1} \\
2 \\
(\mathrm{~Hz}) \\
\end{array}$ & $\begin{array}{l}\mathrm{P}_{2} * 10^{12} \\
(\mathrm{~Hz})\end{array}$ & $\mathrm{C}$ & $\begin{array}{l}\Omega_{2} / \\
\Omega_{1}\end{array}$ & $\begin{array}{l}\mathrm{T}_{2} / \\
\mathrm{T}_{1}\end{array}$ \\
\hline 8.65 & 8.65 & 4.33 & 4.33 & $\begin{array}{l}0.02 \\
6\end{array}$ & 0.5 & $\begin{array}{l}0.01 \\
2\end{array}$ \\
\hline 6.49 & 6.49 & 5.22 & 5.22 & $\begin{array}{l}0.03 \\
2\end{array}$ & 0.8 & 0.12 \\
\hline 5.85 & 5.85 & 5.85 & 5.85 & $\begin{array}{l}0.03 \\
1\end{array}$ & 1 & \\
\hline 5.20 & 5.20 & 6.49 & 6.49 & $\begin{array}{l}0.03 \\
2\end{array}$ & 1.2 & 0.08 \\
\hline 5.77 & 5.77 & 8.65 & 8.65 & $\begin{array}{l}0.01 \\
4\end{array}$ & 1.5 & $\begin{array}{l}0.00 \\
12\end{array}$ \\
\hline
\end{tabular}

$\beta_{1}=0.01$ and $\beta_{2}=0.007[6]$

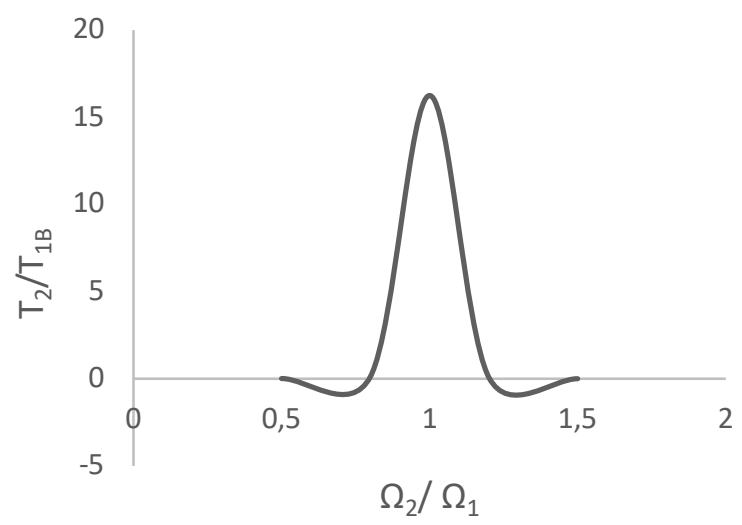

Fig. 6: The ratios of the kinetic energies against frequencies

Consequently, the ratio of kinetic energies will have its maximum value when the two frequencies are equal.

\section{Application of Power Flow in Three Coupled Oscillators to One Dimensional Crystal}

The three masses and four springs system is used as a model to describe the interactions between atoms in a one 
dimensional crystal. The three masses are considered as atoms with identical masses and the forces bonding the atoms represent the coupling. The atoms are connected to each other and to the lattice of the crystal where the forces involved are spring like forces and the atoms are constrained to move in one dimension.

The derived equations of motions are applied to describe the motions of atoms in the crystal which shows that the atoms are collinear with each other. If one of these masses is varied, point defects come into play. Consequently, the different mass system represents a foreign atom in the crystal (substitution impurity) which replaces the position of an original atom in the lattice.

The equations of motion of the system is now used to describe the effect of this substitution impurity in a crystal lattice. The generalized expressions for the three masses and four spring's system can be used to determine the frequency of different systems by varying the coefficient of the middle oscillator $(n)$. However, if $n$ is zero the system reduces to two coupled harmonic oscillators, if $n$ is one the system represents a one dimensional crystal of identical masses and otherwise it represents a crystal exhibiting substitution impurity.

The normal modes obtained for the system of identical masses corresponds to that of Ryan et al., where the oscillators are vertically aligned [8]. The equations of motion of a single oscillator also corresponds to that of Newland et al [3]. Hence, from the derived equations of motion of the two systems, a general equation of motion of an $i^{\text {th }}$ oscillator with a coupling parameter $k$ and a mass $m_{i}$ can be written as:

$m_{i} \ddot{q}_{i}+c_{i} \dot{q}_{i}+\Omega_{i}^{2} q_{i}=f_{i}(t)+k q_{j}$

where $j$ represents the number of oscillators coupled to the $i^{\text {th }}$ oscillator such that $j=1,2,3 \ldots$

In Fig. 3 and 5 the power flow of the two systems decreases exponentially with increase in frequency to an asymptotic value (zero). This indicates that the frequency reaches some threshold value for which the atom acquires enough kinetic energy to break from the bond. Hence, the rate of energy transfers between the oscillator and other oscillators approaches zero. The process can be compared to the photoelectric effect of Albert Einstein in which an electron is emitted from a metal surface when it acquires a threshold frequency (Hugh et al) but in this case an atom is considered and the energy required is known as the cohesive energy (Soren and Peter et al) [5, 9]. Comparatively, the power flow of identical systems is higher than that of the varied middle mass system and thus it needs higher cohesive energy.

From the power flow of the systems, it is observed that atoms acting as substitution impurities are easily removed compared with the atoms making up the crystal due to the lower value of frequency. Moreover, same atoms in a crystal exhibit covalent bond (Natasha et al) while foreign atoms do not share the same amount of electrons and hence exhibit polar covalent bond (Berg et al) with the atoms making up the crystal. Covalent bonds are stronger than polar covalent bonds (Silberg et al) and as a result higher cohesive energy is needed [10-12].

The ratio of the kinetic energies against frequencies for the two systems has its maximum value when the two systems exhibit synchronization which corresponds to that of Newland (1965) for two harmonic oscillators [3].

\section{CONCLUSION}

In this article, general equations representing the frequency of vibrations of a one dimensional crystal are derived and they were used to obtain three different frequencies for two cases (a system of identical masses and system of varied middle mass). The resulting frequencies were used to obtain the normal coordinates as well as the equation of motion in terms of the generalized coordinates. The power flow is calculated under white noise excitation and the results were used to describe the effects of substitution impurities in a one dimensional crystal. It is shown that the presence of a foreign atom tends to distort the periodicity of the lattice structure and thus alters the mechanical and thermal characteristics of a crystal.

There is need to expand this work by using the power flow to obtain the power spectral density of the two systems and observe how the power is distributed over frequency. The results can be useful in revealing the effects of substitution impurities on the spectral density of a one dimensional crystal. Also, different masses with different spring constants can be studied as results can be used to describe the effects of different bond types in a crystal.

\section{REFERENCES}

[1] Frank L. H. W. Coupled Oscillations. Department of Physics and Astronomy, University of Rochester, New York 14627, USA: Physics 235 lecture note, 2013, ch. 12 .

[2] Ndikilar C. E, Analytical Mechanics, ABU Press, Zaria, 2019, ch. 8, pp. 259-264

[3] Newland D.E, "Calculation of Power Flow between Coupled Oscillators”. F. Sound. Vib. vol. 3, no.3, pp. 262-276, 1965

[4] Erik L. (April 18, 2018). Oscillators - a simple introduction.348 Technical University of Denmark, DK2800 Kongens Lyngby, Denmark. [Online] Available: http://orbit.dtu.dk

[5] Hugh D. Y, Roger A.F and Lewis A. F. University Physics with Modern Physics, 13 ${ }^{\text {th }}$ edition. Addison Wisley. San Fransisco, 2012.

[6] Winter. Coupled oscillators and normal modes. PC 235 lecture note, 2013, ch.12.

[7] Chifu E. Ndikilar, Sumayya I. Saleh, Hafeez Y. Hafeez, Lawan S. Taura. "Analytical Calculation of Power Flow between Two Weakly Coupled Pendula and Its Application to an Optical Switch" International Journal of Theoretical and Mathematical Physics, vol. 9, no. 2, pp. 25-35, 2019.

[8] Ryan G. "Direct observation of normal modes in coupled oscillators". American Journal of Physics, pp. 71-87, 2003.

[9] Soren P. B and Peter D. H. Essentials of chemistry, $2^{\text {nd }}$ edition, Ventus publishing Aps, 2010.

[10] Natasha G. (August, 2018). Types of bonding in crystals. [Online] Available: http://sciencing.com

[11] Silberg M. S. Principles of general chemistry, $2^{\text {nd }}$ edition. McGraw Hill publishing company, 2010.

[12] Berg J., Tymoczko J. and Strayer L. Biochemistry, $6^{\text {th }}$ edition. W.H Freeman and company, 2007.

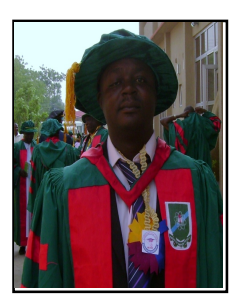

Chifu E. Ndikilar was born in Shisong, Cameroon. He obtained a Doctorate degree in Theoretical Physics from MAUTECH Yola, Nigeria in 2011, Masters Degree in Applied Physics from University of Jos, Nigeria in 2007 and a Bachelors Degree in Physics from the University of Buea, Cameroon in 2002.

He has been a university lecturer for more than a decade and became a Professor in 2016. He is an active researcher of gravitation and theoretical astrophysics. Some of his other research interests include ab initio quantum computations and boundary value problems. He has over fifteen conference presentations \& proceedings and more than 70 articles in peer reviewed journals. He has equally published three books and has edited one book. He is a currently a member of the Editorial Board of Progress in Physics Journal and the Editorin-Chief of Dutse Journal of Pure and Applied Sciences.

Prof. Ndikilar is a member of International Association of Mathematical Physics (MIAMP), Nigerian Institute of Physics (MNIP) and Nigerian Association of Mathematical Physics (MNAMP). He is currently the Deputy 


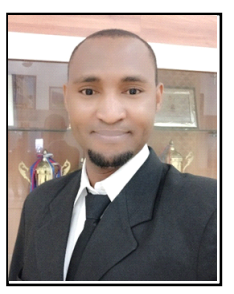

Hafeez Yusuf Hafeez was born in Kano State, Nigeria. He obtained a Doctorate degree in Physics and Nanotechnology from SRM Institute of Science and Technology, India in 2018, Masters Degree in Theoretical Physics from African University of Science and Technology Abuja, Nigeria in 2013 and a Bachelors Degree in Physics from the Bayero University Kano, Nigeria in 2010.

He has been a university lecturer for more than eight years. He is an active researcher of Solar Fuel (i.e. Hydrogen Fuel). Some of his other research interests include Photocatalytic Hydrogen production via water-splitting. He has over ten conference presentations \& proceedings and more than 40 articles in peer reviewed journals. He has equally published one-chapter book. He is a currently a reviewer in Dutse Journal of Pure and Applied Sciences.

Dr. Hafeez is a member of African Material Research Society (MAMRS), Indian Association of Science (MAIS), Nigerian Institute of Physics (MNIP) and Nigerian Association of Mathematical Physics (MNAMP).

Dean, Faculty of Science and also the Director, Directorate of Research and Development.

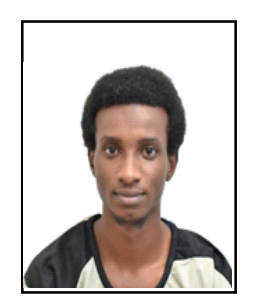

Sofwan Ibrahim Saleh was born in Kano State, Nigeria, in 1994. He received the B.Sc. degree in physics from the University of Sule Lamido (SLU), Kafin Hausa, Jigawa State, Nigeria, in 2018.

In 2015, he joined in the Active Citizen program established by the British council to promote girls child education as a member. He performed an internship program in the year of 2017 for a period of six months in Huawei technologies which he was attached to field maintenance engineers.

Mr. Sofwan carried out his National Youth Service Corps from November 2017 to October 2018 at Iqra Model School as a tutor in Kaduna state, Nigeria. He is currently pursuing his master's degree in physics at the University of Mewar, Chittorgarh, Rajasthan, India.

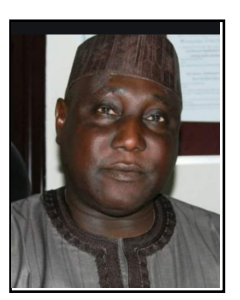

Lawan S. Taura was born in Taura, Jigawa State, Nigeria. He obtained a Doctorate degree in Solid State Physics from Bayero University Kano, Nigeria in 2009, Masters Degree in Solid State Physics from Ahmadu Bello University, Zaria, Nigeria in 1998 and a Bachelors Degree in Physics from the Bayero University Kano, Nigeria in 1990.

He has been a university lecturer for more than a decade and became a Professor in 2013. He specialized in Solid State Physics. Some of his other research interests include ab initio quantum computations and Relativity. He has over twenty conference presentations \& proceedings and more than 50 articles in peer reviewed journals. He is a currently a Vice Chancellor of Sule Lamido University Kafin Hausa, Jigawa State- Nigeria and a member of the Dutse Journal of Pure and Applied Sciences.

Dr. Taura is a member of International Association of Mathematical Physics (MIAMP), Nigerian Institute of Physics (MNIP) and Nigerian Association of Mathematical Physics (MNAMP). 